\title{
Determination of ASI and THIV parameters based on the results of experimental and numerical research in relation to EU standards
}

\author{
Michat Stopel ${ }^{1 *}$, \\ ${ }^{1}$ UTP University of Science and Technology, Al. prof. S. Kaliskiego 7, 85-796 Bydgoszcz, Poland
}

\begin{abstract}
Determining the values of ASI (Acceleration Severity Index) and THIV (Theoretical Head Impact Velocity) parameters during tests allows you to assign an appropriate class for a given type of object to determine the safety level and to give the CE marking. The paper presents the methodology for determining these parameters based on the EN 1317-1 and EN 12767 standards. The paper also presents a tool created with the use of the Python programming language, which, based on the results of experimental tests or the results of numerical calculations, allows to determine the ASI and THIV values. The values of key parameters from the point of view of normative tests were calculated based on the results of experimental tests of the road sign supporting mast and numerical analysis carried out for the same case using the Finite Element Method and LS-Dyna software, following the EN 12767 standard.
\end{abstract}

\section{Introduction}

In accordance with World Health Organization, over a million people are being killed due to road accidents every year. Therefore, some safety mechanisms have to be introduced to provide a proper level of safety for vehicle's occupants. Usually, two types of safety mechanisms can be distinguished: primary (active) and secondary (passive) [1,2]. The first one focuses on preventing an accident from happening. Examples of such solutions among many

are:

AEB - Automatic Emergency Breaking, FCW - Forward Collision Warning, or LDW - Lane Departure Warning. The aim of the passive safety solutions is primarily to minimize the injures severity resulting from road accidents [3]. Nowadays road barriers and passively safe supporting structures fulfil this function [4]. Therefore, for those objects, which constitute road restrictions, it is necessary to carry out crash tests by an accredited body in accordance with the standard appropriate for a given type of object to assign an appropriate safety class. In Europe, there are two important regulations for passive safety objects. EN 1317 "Road restraint systems" [5] - for road barriers and EN 12767 "Passive safety of support structures for road equipment" [6] - for objects like gantries and support masts. Those regulations define the requirements that the tested objects should meet in order to qualify them to the

* Corresponding author: michal.stopel@utp.edu.pl 
appropriate safety class. Both standards specify safety classes based on vehicle speed reduction and such parameters as Acceleration Severity Index (ASI) and Theoretical Head Impact Velocity (THIV). In this paper, the ASI and THIV parameters are explained against the background of the supporting mast crash test in accordance with EN 12767 standard. Additionally, a tool was presented that allows determining the values of these parameters based on both experimental and numerical results.

\section{Materials and methods}

\subsection{Test object}

The tested object was the family of the road signs supporting masts. The family in total consisted of four masts of different sizes. All masts may be considered as a family due to the fact that they were built with the same material, based on the same project, and follow the same mechanism of parts separation. In accordance with EN 12767, there is no need to test experimentally all members of the family of products. Therefore, only the smallest and the largest mast were selected for the crash tests. Figure 1 depicts both masts. The smallest was $4575 \mathrm{~mm}$ tall and could bear a sign board with an area of $2.25 \mathrm{~m}^{2}$. The total mass of the mast with the sign board was $70 \mathrm{~kg}$. The second tested mast was $7060 \mathrm{~mm}$ tall, could bear a sign board with an area of $9.7 \mathrm{~m}^{2}$ and its total weight was $430 \mathrm{~kg}$.

\section{b)}

a)
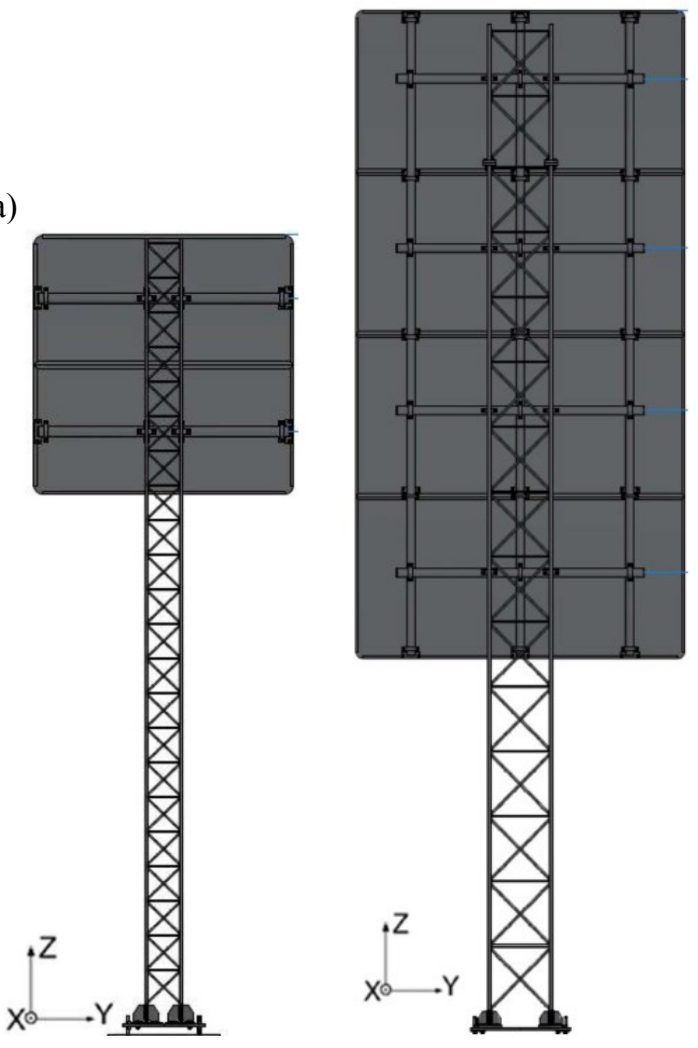

Fig. 1. Tested masts a) $2.5 \mathrm{~m}^{2}$, b) $9.7 \mathrm{~m}^{2}$ 


\subsection{EN 12767 safety class requirements}

EU standard EN 12767 specifies a method for assessing the passive safety properties of supporting structures such as lighting poles, signposts, supports for road signs, structural elements, foundations, and any other components used as roadside equipment. This document is a common basis for crash testing of structural elements supporting road equipment, it does not include requirements and testing methods for road restraint systems. Conformity assessment requirements for restraint systems like safety barriers, crash cushions are specified in EN 1317 standard. Both documents classify the construction to a proper safety class accordingly to the values of ASI and THIV parameters and vehicle velocity loss, calculated from experimental data. Further details will be discussed on the example of EN 12767.

The EN 12767 standard states that crash tests should be carried out in the range of two velocities and that the choice of velocity classes are 50,70 , and $100 \mathrm{~km} \cdot \mathrm{h}^{-1}$. The velocity class should be selected appropriately to the place of application of the designed structure. For each of the selected velocity classes, the level of impact energy absorption is determined separately. For the speed class of $100 \mathrm{~km} \cdot \mathrm{h}^{-1}$, which was selected for the constructions in question, there should be crash tests at 35 and $100 \mathrm{~km} \cdot \mathrm{h}^{-1}$ carried out.

The standard defines three classes of energy absorption: NE - no energy absorption, LE low level of absorption, and HE - high level of absorption. Contrary to what might seem at first glance, the highest level of safety according to the provisions of the standard is ensured by structures that do not absorb the impact energy. They should be the smallest obstacle for the vehicle, which is equipped with active passenger safety systems. It is the opposite assumption in relation to road restraint barriers, which are to prevent vehicles from leaving the track. Table 1 represents the dependence of the energy absorption class to the vehicle velocity after the crash with the structure for the velocity class of $100 \mathrm{~km} \cdot \mathrm{h}^{-1}$.

Table 1. Energy absorption class for the velocity class of $100 \mathrm{~km} \cdot \mathrm{h}^{-1}$

\begin{tabular}{|c|c|}
\hline $\begin{array}{c}\text { Velocity class, } \\
\mathrm{km} \cdot \mathrm{h}-1\end{array}$ & 100 \\
\hline $\begin{array}{c}\text { Energy absorption } \\
\text { class }\end{array}$ & $\begin{array}{c}\text { Output velocity, } \mathrm{ve}, \\
\mathrm{km} \cdot \mathrm{h}-1\end{array}$ \\
\hline $\mathrm{HE}$ & $5<\mathrm{ve} \leq 50$ \\
\hline $\mathrm{LE}$ & $50<\mathrm{ve} \leq 70$ \\
\hline $\mathrm{NE}$ & $70<\mathrm{ve} \leq 100$ \\
\hline
\end{tabular}

Vehicle occupants' safety class is related to the probability of injuries occur as a result of an accident. Indicators that allow approximating the level of injuries that may occur are mentioned before ASI and THIV. Occupants' safety classes in relation to those parameters are presented in Table 2 . 
Table 2. Vehicle occupant's safety class for the velocity class of $100 \mathrm{~km} \cdot \mathrm{h}^{-1}$

\begin{tabular}{|c|c|c|c|c|c|}
\hline \multirow{4}{*}{$\begin{array}{l}\text { Energy } \\
\text { absorption } \\
\text { class }\end{array}$} & \multirow{4}{*}{$\begin{array}{c}\text { Vehicle } \\
\text { occupant's } \\
\text { safety class }\end{array}$} & \multicolumn{4}{|c|}{ Velocity } \\
\hline & & \multicolumn{2}{|c|}{$\begin{array}{l}\text { Test with low } \\
\text { impact velocity } \\
35 \mathrm{~km} \cdot \mathrm{h}-1\end{array}$} & \multicolumn{2}{|c|}{$\begin{array}{l}\text { Test with high } \\
\text { impact velocity } \\
100 \mathrm{~km} \cdot \mathrm{h}-1\end{array}$} \\
\hline & & \multicolumn{2}{|c|}{ Upper bound values } & \multicolumn{2}{|c|}{ Upper bound values } \\
\hline & & ASI & THVI, $\mathrm{km} \cdot \mathrm{h}-1$ & ASI & THVI, km·h-1 \\
\hline \multirow{4}{*}{$\mathrm{HE} / \mathrm{LE} / \mathrm{NE}$} & $\mathrm{E}$ & 1 & 27 & 1,4 & 44 \\
\hline & $\mathrm{D}$ & 1 & 27 & 1,2 & 33 \\
\hline & $\mathrm{C}$ & 1 & 27 & 1 & 27 \\
\hline & B & 0,6 & 11 & 0,6 & 11 \\
\hline $\mathrm{NE}$ & A & \multicolumn{2}{|r|}{---} & \multicolumn{2}{|r|}{---} \\
\hline
\end{tabular}

\subsection{Acceleration Severity Index}

The value of Acceleration Severity Index - ASI is determined by the equation (1), wherein the numerators of individual terms of the equation there are components of the vehicle acceleration and in the denominator the corresponding acceleration limits $\left(\widehat{a_{x}}=12 \mathrm{~g}, \widehat{a_{y}}=\right.$ $9 g, \widehat{a_{z}}=10 \mathrm{~g}$ ). Accelerometers used for measuring the acceleration components should be placed close to the vehicle's centre of gravity. The ASI is a single maximum value of collected data (2).

$$
\begin{aligned}
& A S I(t)=\sqrt{\left(\frac{a_{x}}{\widehat{a_{x}}}\right)^{2}+\left(\frac{a_{y}}{\widehat{a_{y}}}\right)^{2}+\left(\frac{a_{z}}{\widehat{a_{z}}}\right)^{2}} \\
& A S I=\max [A S I(t)]
\end{aligned}
$$

\subsection{Theoretical Head Impact Velocity}

The value of Theoretical Head Impact Velocity - THIV, can be obtained computationally on the basis of the recorded acceleration values of the vehicle since the assumption is made that the occupant head velocity at the time of impact is the same as vehicle velocity in the horizontal plane. THIV is then defined as (3):

$$
T H I V=\left[\mathrm{V}_{\text {head } x}{ }^{2}(T)+\mathrm{V}_{\text {head }} y^{2}(T)\right]
$$

where $V_{\text {head } x}, V_{\text {head } y}$ are the head velocities in longitudinal and transverse directions of the vehicle coordinate system, $C_{\text {vehicle }}, \mathrm{T}$ is the time of flight which corresponds to the absolute value of theoretical head displacement equal to $600 \mathrm{~mm}$ in the $\mathrm{x}$-direction or $300 \mathrm{~mm}$ in the y-direction. 
To get the values of $V_{\text {head } x}, V_{\text {head } y}$, a series of transformations should be done. At first, the acceleration of the vehicle coordinate system should be calculated with the equation (4):

$$
\left\{\begin{array}{l}
\ddot{X}_{\text {vehicle }}=a_{\text {vehicle } x} \cdot \cos \theta-a_{\text {vehicle } y} \cdot \sin \theta \\
\ddot{Y}_{\text {vehicle }}=a_{\text {vehicle } x} \cdot \cos \theta-a_{\text {vehicle } y} \cdot \sin \theta
\end{array}\right.
$$

Then the head displacement can be calculated from (5):

$$
\left\{\begin{array}{c}
x_{\text {head }}=\left(x_{0}-X_{\text {vehicle }}\right) \cdot \cos \theta-\left(y_{0}-Y_{\text {vehicle }}\right) \cdot \sin \theta \\
y_{\text {head }}=-\left(x_{0}-X_{\text {vehicle }}\right) \cdot \sin \theta+\left(y_{0}-Y_{\text {vehicle }}\right) \cdot \cos \theta
\end{array}\right.
$$

The, $X_{\text {vehicle }}, Y_{\text {vehicle }}$ are the displacement of the vehicle coordinate system acquired by double integration of equation 4.

Values of the head velocity components are then calculated as:

$$
\left\{\begin{array}{l}
v_{\text {head } x}=-\dot{X}_{\text {vehicle }} \cdot \cos \theta-\dot{Y}_{\text {vehicle }} \cdot \sin \theta+y_{\text {head }} \cdot \dot{\theta} \\
v_{\text {head } y}=-\dot{X}_{\text {vehicle }} \cdot \sin \theta-\dot{Y}_{\text {vehicle }} \cdot \cos \theta-x_{\text {head }} \cdot \dot{\theta}
\end{array}\right.
$$

\section{Python tool for ASI and THIV calculations}

In order to determine the ASI and THIV parameters, based on the previously presented formulas (1-5), an application was developed using the Python programming language. Presented code may be also a part of a future software that will allow to validate and change parameters, such as Johnson-Cook's material model constants [7], of finite element model created with the use of LS-Dyna *. k, based on the results of experimental research.

Selected program lines are presented in this chapter.

Loading of data recorded by accelerometers:

df0 $=$ pd.read_csv(data_folder + File, sep=';', skiprows $=1)$

dane $0=$ df0.values

$\mathrm{t}=$ dane $0[:, 0]$

x_acc $=$ dane $0[:, 1]$

y_acc $=$ dane $0[:, 2]$

z_acc $=$ dane $0[:, 3]$

yaw $=$ dane $0[:, 4]$

roll $=$ dane $0[:, 5]$

pitch $=$ dane $0[:, 6]$

Entry data was filtered using fourth order low-pass Butterworth digital filter with a $3 \mathrm{~dB}$ cutoff frequency of $300 \mathrm{~Hz}$ (Channel Frequency Class 180) or $100 \mathrm{~Hz}$ (CFC 60):

sos $=$ signal.butter $(4, \mathrm{fc}$, 'low', analog=False, output='sos',fs $=$ fsd $)$

Axis_data_filt $=$ signal.sosfilt(sos, Axis_data)

There is filtered and unfiltered data for the $\mathrm{x}$-axis acceleration depicted in figure 2 . 


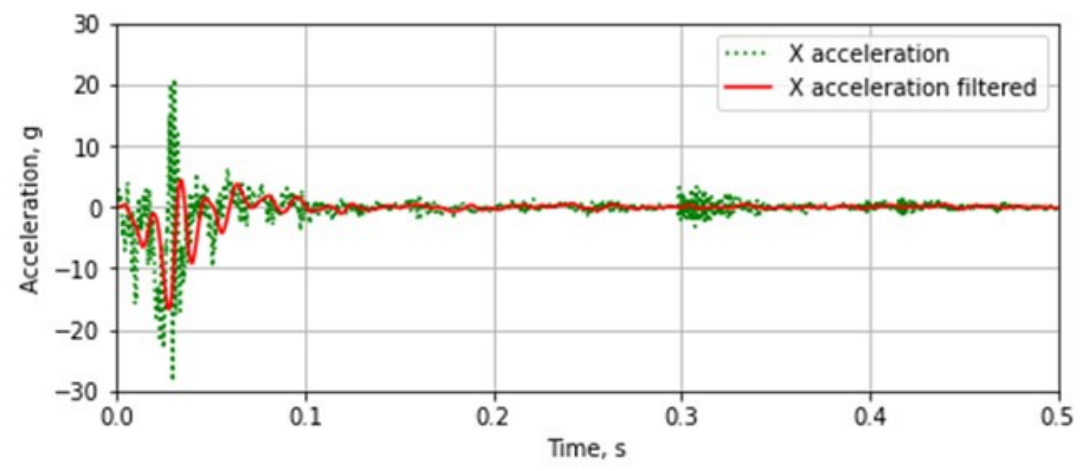

Fig. 2. Filtered and unfiltered data of car acceleration in the x-axis.

To calculate ASI value accordingly to equation 1 the 50-ms moving average is computed:

$\mathrm{d} 0=$ pd.Series $\left(\mathrm{x} \_\mathrm{acc}\right)$

$\mathrm{d} 1=$ pd.Series $\left(\mathrm{y} \_\mathrm{acc}\right)$

$\mathrm{d} 2=$ pd.Series $\left(\mathrm{z} \_\right.$acc $)$

d0_mva $=$ d0.rolling $($ window $=500) \cdot \operatorname{mean}()$

d1_mva $=$ d1.rolling $($ window $=500) \cdot \operatorname{mean}()$

$\mathrm{d} 2 \_$mva $=\mathrm{d} 1$.rolling $($ window $=500) \cdot \operatorname{mean}()$

Then the values of individual members of equation 3, corresponding to the $\mathrm{x}, \mathrm{y}$, and $\mathrm{z}$ acceleration components are determined:

$\mathrm{D} 0=\mathrm{d} 0 \_\mathrm{mva} / 12 / 9.81$

$\mathrm{D} 1=\mathrm{d} 1 \_\mathrm{mva} / 9 / 9.81$

$\mathrm{D} 2=\mathrm{d} 2 \_\mathrm{mva} / 10 / 9.81$

Subsequently equation 1 in python code is reduced to:

$\mathrm{ASI}=\mathrm{np} . \operatorname{sqrt}(\mathrm{D} 0 * * 2+\mathrm{D} 1 * * 2+\mathrm{D} 2 * * 2)$

Figure 3 depicts a graphic interpretation of the ASI parameter.

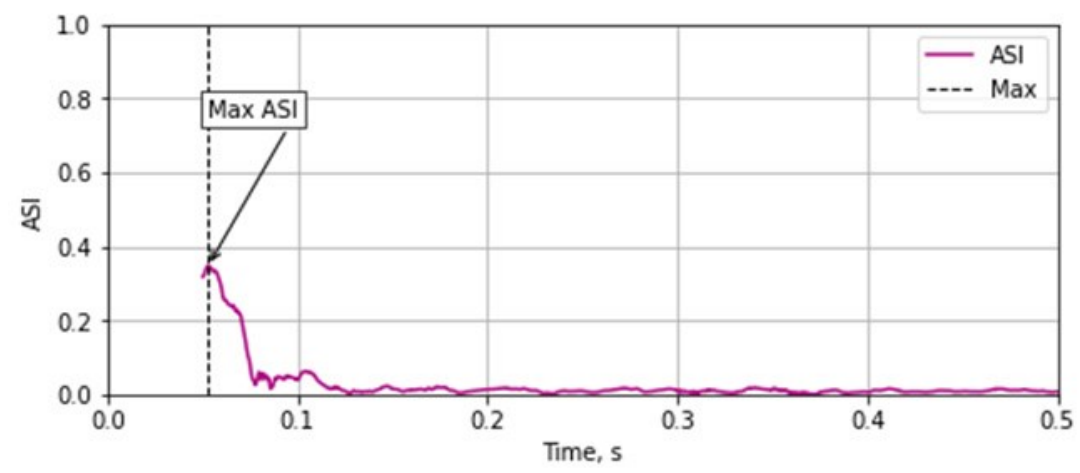

Fig. 3. ASI parameter vs time 
To calculate THIV value entry data of yaw velocity have to be integrated to get yaw angle $\theta$ :

yaw_rad $=$ np.deg2rad(yaw)

$\mathrm{FI}=$ intg.cumtrapz(yaw_rad, $\mathrm{t}$, initial $=0.0)$

Then the vehicle acceleration can be calculated accordingly to equation 4 , vehicle velocity by the integration of eq. 4 , and the vehicle displacement by double integration of eq. 4 :

Xcc=x_acc_filt*3.6*np.cos(FI)-y_acc_filt*3.6*np.sin(FI)

Ycc=x_acc_filt*3.6*np.sin(FI)-y_acc_filt*3.6*np.cos(FI)

$\mathrm{Xc}=($ intg. $\operatorname{cumtrapz}(\mathrm{Xcc}, \mathrm{t}$, initial $=0.0))$

$\mathrm{Yc}=($ intg.cumtrapz $(\mathrm{Ycc}, \mathrm{t}$, initial $=0.0))$

$\mathrm{X}=($ intg.cumtrapz $(\mathrm{Xc}, \mathrm{t}$, initial $=0.0))$

$\mathrm{Y}=($ intg.cumtrapz $(\mathrm{Yc}, \mathrm{t}$, initial $=0.0))$

Now the head displacement (5) and the value of theoretical head velocity in $\mathrm{x}$ and $\mathrm{y}$ direction (6) can be determined:

$$
\begin{aligned}
& \mathrm{x}=(0-\mathrm{X}) * n p \cdot \cos (\mathrm{FI})-(0-\mathrm{Y}) * n p \cdot \sin (\mathrm{FI}) \\
& \mathrm{y}=-(0-\mathrm{X}) * n p \cdot \sin (\mathrm{FI})+(0-\mathrm{Y}) * n p \cdot \cos (\mathrm{FI}) \\
& \text { vxc=-Xc*np.cos(FI)-Yc*np.sin(FI)+y*yaw_rad } \\
& \text { vyc=-Xc*np.sin(FI)-Yc*np.cos(FI)-x*yaw_rad }
\end{aligned}
$$

Subsequently equation 3 in python code is reduced to:

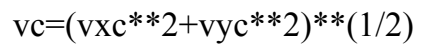

Figure 4 depicts a graphic interpretation of the THIV parameter.

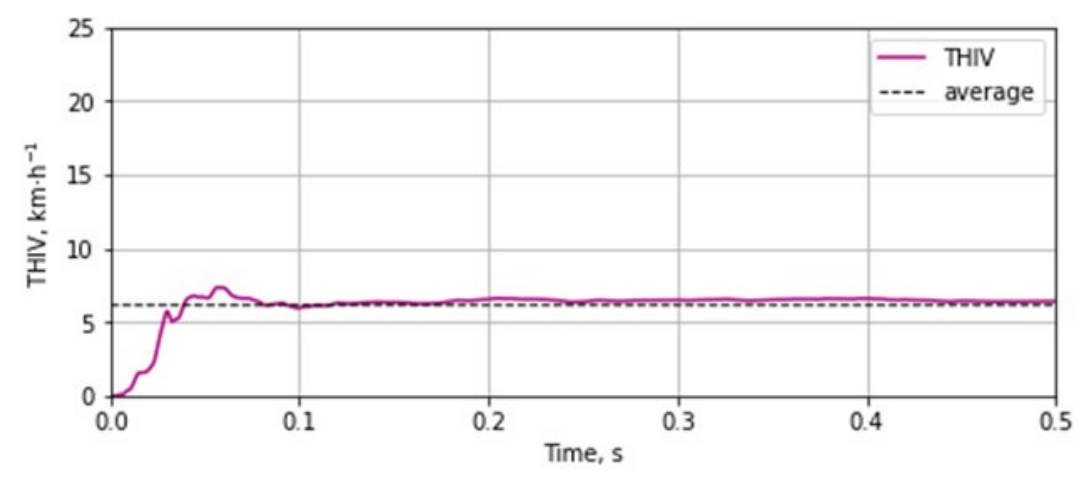

Fig. 4. THIV value vs time 


\section{Results}

\subsection{Experimental test}

Experimental tests were carried out for two family members depicted in Figure 5. The tests were carried out in an accredited laboratory, and they were tests certifying the structure based on the mentioned standard. In total, four crash tests were carried out, i.e., pair for each structure: a low-velocity test at an initial vehicle velocity of $35 \mathrm{~km} \cdot \mathrm{h}-1$ and a high-velocity test of $100 \mathrm{~km} \cdot \mathrm{h}-1$.

During the experiment, quantitative parameters such as vehicle velocity loss or linear and angular accelerations were recorded. Accelerometers were located in the centre of gravity of the vehicle. The selection of the vehicle and the trajectory were selected based on the normative guidelines. Figure 5 depicts the result of the crash test carried out at a speed of 100 $\mathrm{km} \cdot \mathrm{h}^{-1}$ for the small mast.

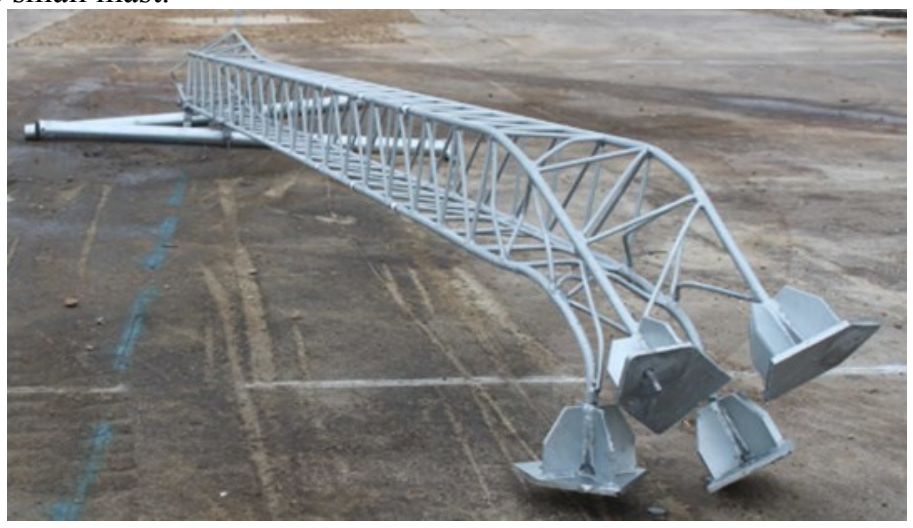

Fig. 5. Small mast after crash test with the vehicle moving at the initial speed of $100 \mathrm{~km}$

To calculate the values of the ASI and THIV parameter described Python code was used. As a result of those calculations ASI parameter was determined as 0.35 and THVI as 6.14 $\mathrm{km} \cdot \mathrm{h}-1$. Therefore, the construction was classified as non-energy absorbing (NE) with passenger safety class B.

\subsection{Brief description of numerical calculations}

The performed experimental research will allow to validate the numerical model, previously prepared at the design stage with the LS-Dyna explicit solver, appropriate to solve highly non-linear and dynamic problems [8]. Especially important is to validate the model of part separation due to the failure of the bolts [9]. Therefore, one of the main phenomena to recognize was the distribution of mechanical properties in the bolt cross-section [10]. The finite element model consisted of nearly 72000 elements depending on masts family members and included 6 different material models, 13 contact interfaces, and the calculation time for one case was 15 hours with the use of 11 computing cores. For the calculation of ASI and THIV the same code was used as in experimental tests and the values were determined as 0.26 and $6.1 \mathrm{~km} \cdot \mathrm{h}^{-1}$. A small mast after the crash test is depicted in figure 6 . 


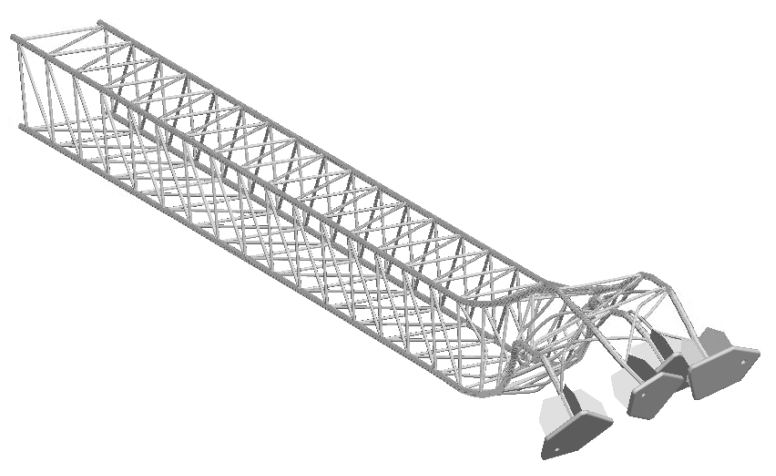

Fig. 6. Small mast after crash test with the vehicle moving at the initial speed of $100 \mathrm{~km}-\mathrm{FEM}$

\section{Conclusions}

The presented tool created with the use of Python programming code proved to be extremely useful in the process of determining ASI and THIV parameters values. It can be simply implemented at the stage of processing the results of experimental tests. It can be also included in the post-processing of numerical simulations and to adjust material constants based on comparison of numerical and experimental results.

\section{References}

1. Cell J., Brandani C., E., Fraschetti S., Chakraverty J., Accident Anal Prev, 133 (2019)

2. Wilde K., Tilsen A., Burzyński S., Witkowski W., On the influence of the acceleration recording time on the calculation of impact severity indexes, in Proceedings of the MATEC Web of Conferences 219, 20-23 April, Gdynia, Poland (2018)

3. Sturt R., Fell C., Int J Crashworthiness, 14:2, 165-172 (2009)

4. Kunc R., Omerović S., Ambroz M., Prebil I., Transp Res Proc, 14, 1305-1314, (2016)

5. EN 1317-1:2010, Road Restraint Systems - Part 1: Terminology and General Criteria for Test Methods (2010)

6. EN 12767:2019, Passive safety of support structures for road equipment - Requirements and test methods (2019)

7. Stopel, M., Skibicki, D., Cichański, A., Mater Test, 60, 10, 974-978 (2018)

8. Skibicki D., Pejkowski Ł., Stopel M., Pol Marit Res, 24, 4, 116-123 (2017)

9. Stopel M., Cichański A., Skibicki D., Modeling of Prestressed Bolt Connection in LsDyna Crash Test Analysis of Road Infrastructure, Proceedings of 23rd International Conference Engineering Mechanics, 15-18 May, Svratka, Czech Republic (2017)

10. Cichański A., Skibicki A., Experimental studies of the mechanical properties distribution in the M16 bolt core, AIP Conference Proceedings 2029, 020011, 4 -6 June, Zakopane, Poland (2018) 